\title{
Resource Combinatorial Optimization for Mobility-aware STDMA MAC Protocols
}

\author{
Siqian Cui*, Yingyuuan Gao, Bin Li and Zhou Lu \\ China Academy of Electronics and Information Technology, Beijing 100041, China. \\ csq_0303@163.com,gyy_0303@163.com, libincaeit@163.com, luzhoucaeit@163.com
}

\begin{abstract}
In this paper, a Mobility Pattern Adaptive Resource Combinatorial Optimization (MPARCO) algorithm is developed for mobility-aware STDMA MAC protocols in multi-hop wireless networks. The underlying problem entails the optimal joint link scheduling combined with the simultaneous assignment of transmit power levels and data rates across active mobile links. We develop a mathematical formulation for maximizing throughput and/or power efficiency under corresponding constrains. Then, we solve the problem with Discrete Dynamic Programming (DDP) depending on different mobility patterns. Finally, based on the solution, we propose our low complexity distribute MPARCO strategy which can be generically embedded into any existing mobility-aware STDMA MAC protocol.
\end{abstract}

Keywords: Energy-efficient, Combinatorial Optimization Problem, Discrete Dynamic Programming.

\section{Introduction}

Our research considers modern mobile services accommodated by multi-hop wireless networks such as a wireless mesh network. A large number of variant STDMA MAC protocols were explored to adapt to users' mobility in such kind of networks [1,2]. To improve the throughput capacity and energy consumption performance of such STDMA MAC protocols, a joint link scheduling combined with the simultaneous assignment of transmit power levels and data rates across mobile active links is strongly demanded. In practice, however, the need to accommodate different mobility patterns makes resource scheduling algorithms very hard to implement and the problem of doing so in a practically viable manner remains unsolved. Accordingly, the primary motivation of this work is to exploit a mobility pattern adaptive resource combinatorial optimization algorithm for mobility-aware STDMA MAC protocols.

\section{Analytical Model}

We use synthetic models to describe the mobility patterns $[3,4]$. Which means that our mobility patterns represent the behaviors of real-world mobile objects. Further, we do not produce the precise description about the direction and magnitude of mobility and just use the Physical Interference Model (PIM) [5] to measure if a successful transmission on $l_{i_{k}}^{j_{k}}$ be broken or not:

$$
\frac{P_{i_{k}}^{j_{k}} G_{i_{k}}^{j_{k}}}{\sum I_{i_{p}}^{j_{k}}+N_{j_{k}}} \geq \gamma\left(r_{i_{k}}^{j_{k}}\right), \quad i_{k} \neq j_{k} \neq i_{p} \neq j_{p}
$$

where $r_{i_{k}}^{j_{k}}$ is the data rate on link $l_{i_{k}}^{j_{k}}$ which can be selected from within the discrete set $\left\{r_{1}, r_{2}, \cdots, r_{m}\right\}$ with $r_{1}<r_{2}<\ldots<r_{m} . P_{i_{k}}^{j_{k}}$ is the power used by the sending node $i_{k}$ and $P_{i_{k}}^{j_{k}} \leq P_{\max } . N_{j_{k}}$ represents the thermal noise on the receiver $j_{k}, \sum I_{i_{p}}^{j_{k}}$ is the interference caused by other simultaneous transmissions and $\gamma\left(r_{i_{k}}^{j_{k}}\right)$ is the pre-specified SINR threshold [6]. Finally, $G_{i_{k}}^{j_{k}}$ represents the path loss from node $i_{k}$ to node $j_{k}$. We consider noisy channels in which the transmitter does not have perfect channel state information (CSI) and just calculate $G_{i k}^{j_{k}}$ according to the radio propagation model $G_{i_{k}}^{j_{k}}=1 / d^{\theta}$ in which $\theta$ is the path loss exponent ranging from 2 when in a line of sight free space environment to 4 when in an indoor 
environment [7]. We further capture the effects of wireless channel such as shadowing and the effects of mobility by the expressions of Packet Error Rate (PER) [8].

\section{Power and Rate Relationship in mobile Simultaneous Transmission Scenarios}

In this section, we explore the relationship between achievable data rates and corresponding transmission power levels in mobile simultaneous transmission environments. Similar to the approach of [9] and many other papers, the mobility patterns of an LCA are approximately divided into Weak Mobility (WM) and Strong Mobility (SM) in our research. Our goal is maximizing the throughput of a given time slot in strong mobility and maximizing the energy efficiency in weak mobility. Our goal can be described by the following optimization problem.

$$
\begin{aligned}
& \underset{P_{i_{k}}^{j_{k}}}{\max } \sum r_{i_{k}}^{j_{k}} \text { or } \max _{r_{i_{k}}^{j_{k}}} \frac{\sum r_{i_{k}}^{j_{k}}}{\sum P_{i_{k}}^{j_{k}}} \\
& \text { s.t. }\left\{\begin{array}{l}
r_{i_{k}}^{j_{k}} \in\left\{r_{1}, \cdots, r_{m}\right\}, \quad r_{1}<r_{2}<\ldots<r_{m} \\
0 \leq P_{i_{k}}^{j_{k}} \leq P_{\max } \\
R_{i_{k}}^{j_{k}} \leq r_{i_{k}}^{j_{k}} \leq r_{m}, \quad i_{k} \neq j_{k} \\
\frac{P_{i_{k}}^{j_{k}} G_{i_{k}}^{j_{k}}}{\sum_{i_{i}}^{j_{k}}+N_{j_{k}}} \geq \gamma\left(r_{i_{k}}^{j_{k}}\right), \quad i_{k} \neq j_{k} \neq i_{p} \neq j_{p} \\
\gamma(r)=f_{k}(r) \in\left\{f_{1}(r), f_{2}(r), \cdots, f_{n}(r)\right\}
\end{array}\right.
\end{aligned}
$$

The last constraint illustrates the relationship between the SINR threshold $\gamma(r)$ and the corresponding data rate $r$. It can be expressed by the polynomial below.

$$
\gamma(r)=\lambda_{n} r^{n}+\lambda_{n-1} r^{n-1}+\ldots+\lambda_{2} r^{2}+\lambda_{1} r+\lambda_{0}
$$

In the equation above, $\lambda_{n}, \lambda_{n-1}, \ldots, \lambda_{0} \in R^{+}$and can be valued different quantities combination depending on the mobility patterns. Under simultaneous transmission environments, based on (1), the available data rate of a link depends on both that link's own transmission power level as well as the transmission power levels of all other simultaneous transmissions. To explore the exact relationship between these parameters, we define and prove the following theorems.

Definition 1: We define a transmission scenario $S(i \rightarrow j)=\left\{i_{1} \rightarrow j_{1}, i_{2} \rightarrow j_{2}, \ldots, i_{k} \rightarrow j_{k}, \ldots, i_{n} \rightarrow j_{n}\right\}$ with links physically move through each other' effective interference range is feasible under power vector $P_{i}^{j}=\left\{P_{i_{1}}^{j_{1}}, P_{i_{2}}^{j_{2}}, \ldots, P_{i_{k}}^{j_{k}}, \ldots, P_{i_{n}}^{j_{n}}\right\}, 0 \leq P_{i_{k}}^{j_{k}} \leq P_{\max }$, if at least one data rate vector $R_{i}^{j}=$ $\left\{r_{i_{1}}^{j_{1}}, r_{i_{2}}^{j_{2}}, \ldots, r_{i_{k}}^{j_{k}}, \ldots, r_{i_{n}}^{j_{n}}\right\}$ is available in the range $R_{i_{k}}^{j_{k}} \leq r_{i_{k}}^{j_{k}} \leq r_{m}, k=1,2, \ldots, n$.

Definition 2: We refer to the data rate vectors of an $n$ successful mobile simultaneous transmission scenario $S(i \rightarrow j)$ which generate the maximized throughput of a slot under current experiencing nodal distribution and mobility pattern as Throughput Optimal Rate Vectors (TORV).

Definition 3: We refer to the power vectors under which an $n$ successful mobile simultaneous transmission scenario $S(i \rightarrow j)$ is feasible which TORV under current experiencing nodal distribution and mobility pattern as Throughput Optimal Power Vectors (TOPV).

Definition 4: We refer to data rate vector $R_{i}^{j}=\left\{R_{i_{1}}^{j_{1}}, R_{i_{2}}^{j_{2}}, \ldots, R_{i_{k}}^{j_{k}}, \ldots, R_{i_{n}}^{j_{n}}\right\}$ of an $n$ successful mobile simultaneous transmission scenario $S(i \rightarrow j)$ as Per Component Lowest Rate Vector (PCLRV).

From (1) and (3), we know that the relationship between the available data rates and corresponding power levels in successful mobile simultaneous transmissions is reversible. Therefore, for an $n$ successful mobile simultaneous transmission scenario with links physically move through each other' effective interference range, the highest energy efficiency is obtained at the PCLRV no matter what the power vector $P_{i}^{j}=$ 
$\left\{P_{i_{1}}^{j_{1}}, P_{i_{2}}^{j_{2}}, \ldots, P_{i_{k}}^{j_{k}}, \ldots, P_{i_{n}}^{j_{n}}\right\}$ is. In other words, for mobile simultaneous transmissions, when under strong mobility pattern, MAC protocols should adopt the TORV and finish the packets on active mobile links as quick as possible to reduce the probability of collisions. On the other hand, when under weak mobility pattern, transmitting packets at PCLRV is more energy efficient. These two theorems provide us with a new perspective in looking for a more appropriate treatment of mobile simultaneous transmissions.

\section{Mobility Pattern Adaptive Resource Combinatorial Optimization Algorithm}

In this section, we present the design and experimental analysis of our MPARCO algorithm.

\subsection{Distribute Optimization Algorithm Design}

Based on the analysis above, our MPARCO may operate in WM mode or SM mode. We take MTDMA for example here to illustrate how MPARCO changes the mechanism of the existing mobilityaware STDMA protocols. Two kinds of control packets are embedded into M-TDMA and called Transmit Requirement (TR) packet and Transmission Permission Information (TPI) packet. TRs are transmitted to the slot owner by the nodes which would like to transmit within that slot when the operation changes from WM mode to SM mode. By sending a TR to the slot owner, a node informs the slot owner about the position of the sender and the receiver. The required $R_{i_{k}}^{j_{k}}$ of the link should be contained in the TR. TPIs are transmitted by the slot owner to inform the calculated rates information to the nodes which are permitted to transmit in SM mode [10].

1) WM mode: After the time slot assignment and clock synchronization upon the startup of M-TDMA protocol, we just assume that the mobility pattern of the LCA is WM and MPARCO works in WM mode. While in WM mode, all active transmitters are allowed to transmit in any time slot with rate equal to the required $R_{i_{k}}^{j_{k}}$ of those links. Further, any of the active transmitters just uses the power level that can support its own transmission under corresponding SNR constraint. However different power margins is left at the receivers according to the mobility pattern of a link. Must be emphasized is that the probability of collisions caused by simultaneous transmissions is small in WM mode. If the influence of the physically move results in a large number of collisions, MPARCO will switch to SM mode which will be introduced in the next subsection. For the given placement of nodes and mobility patterns, the traditional STDMA MAC protocols will exclude each other' transmission. However, if all the senders reduced their transmission power levels such that it would be just enough for their own receivers to capture the signal and do not cause so much interference to the other links then other nodes in the same LCA could also proceed with their transmissions. Such a WM mode would allow for a tighter packing of source destination pairs within an LCA, thereby improving the space reuse and energy efficiency. Fig. 1 shows the motivation of the WM mode.

2) SM mode: We define $N_{t h}$ is the mode switching threshold. The mobility pattern is estimated by counting the number of lost ACKs. If the slot owner misses $N_{t h}$ consecutive ACKs, a notification message will be sent with $T T L=2$ to inform the senders in its LCA to send TRs. To avoid the collisions of the TRs, every sender interested in transmission sets a random timer. As one sender's timer goes off, the associated TR will be sent to inform its intent to transmit to the slot owner. To gather this information, the slot owner listens for transmission requests from the neighbors for a certain short period of time referred to as $\tau$. The listening period $\tau$ is typically set to $3 \%$ percent of the slot duration. In order to statistically guarantee the delivery of transmitted TRs, the random timer $t_{i}$ at node $i$ is set as follows.

$$
t_{i}=t_{0}+r \quad 0 \leq r \leq 0.03 T_{\text {slot }}
$$

where $t_{0}$ represents the time that $N_{t h}$ consecutive ACKs missing are counted and $r$ is a number selected randomly. Upon the receipt of all the TRs, the slot owner begins to evaluate whether a node can be granted permission to simultaneously transmit in its slot. When the slot owner finishes the calculation, it will hand out the results to its neighbors and begin transmitting. The other nodes granted permission to transmit will begin transmitting after receiving TPIs from the slot owner using the optimal data rates as dictated by the slot owner. The steps of the mode switching are show in Fig. 2. 


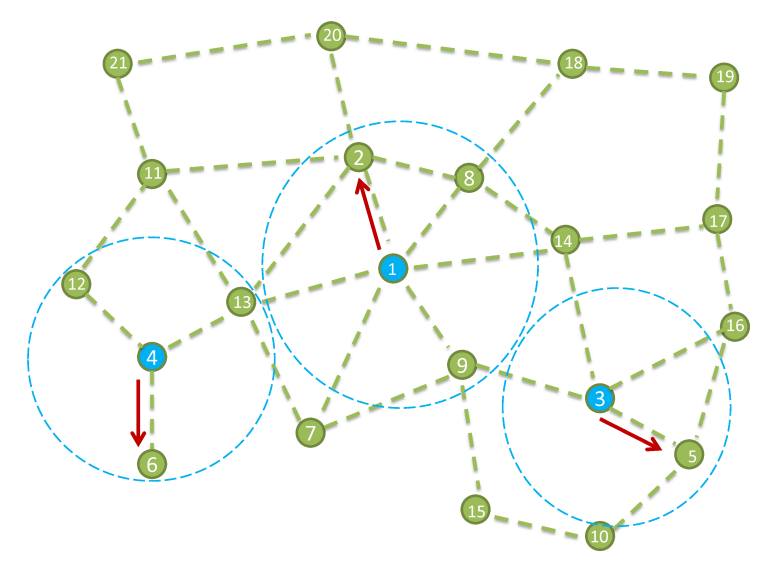

Fig. 1: Motivation of the Weak Mobility (WM) mode.

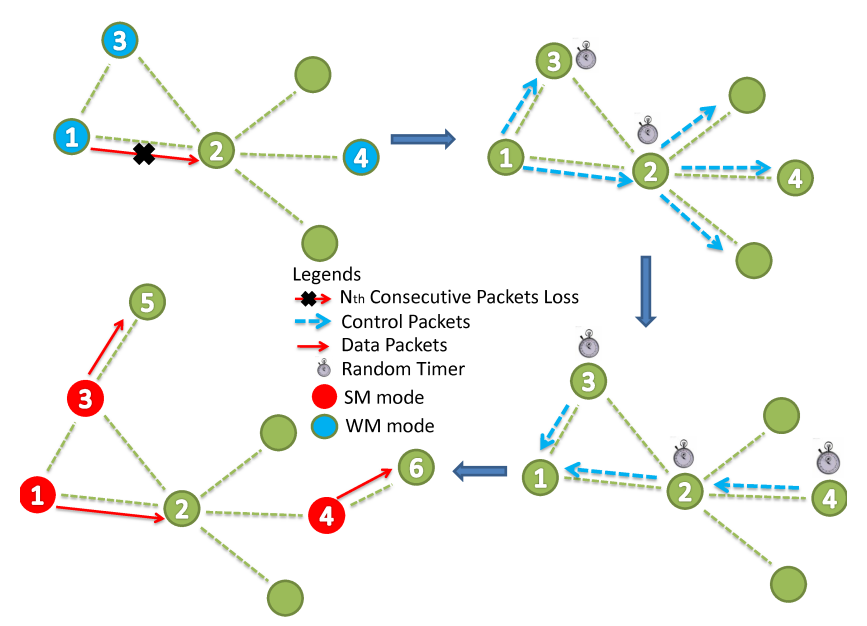

Fig. 2: The steps of the mode switching.

\subsection{Experimental Analysis}

To study the performance effects of our algorithm on mobility-aware STDMA MAC protocols, numerical experiments are carried out in NS3. We use M-TDMA and V-TDMA as original non resource optimization benchmarks and DPRL as a resource optimized STDMA benchmark for evaluating the performance of our algorithm. We refer to M-TDMA and V-TDMA with MPARCO as MRCOM and MRCOV respectively. DPRL is a greedy distributive heuristic resource schedule algorithm which can be used to schedule multiple mobility transmissions iteratively in a given time slot based on the highest $S I N R$ value within the two-hop neighborhood not interfering with existing transmissions. In our experiments, we generate the network topologies by distributing 10 to 20 nodes independently and uniformly in a square area of dimensions $200 \times 200$ square meters. Our simulation program randomly selects links formed by these nodes. Each selected transmitter-receiver pair chooses one of six relative speeds in the set $\left\{s_{1}=5, s_{2}=10, s_{3}=15\right.$, $\left.s_{4}=20, s_{5}=25, s_{6}=30\right\} \mathrm{km} / \mathrm{h}$ and the corresponding $R_{i}^{j}$ is in the set $\left\{r_{1}=1, r_{2}=3, r_{3}=5\right.$, $\left.r_{4}=7, r_{5}=9, r_{6}=11\right\}$ packets/slot respectively. The power margin corresponding to the speed is set to $\lg \sqrt{\text { speed }} \mathrm{dBm}$. We experiment with fixed length data packets of size 64 bytes. The path loss exponent is fixed to 3 . The value of $P_{\max }$ is set to $35 \mathrm{dBm}$ and the average thermal noise $N$ is $-30 \mathrm{dBm}$. The slot length is set to $50 \mathrm{~ms}$ and the operation duration of the MPARCO algorithm is limited to $5 \mathrm{~ms}$. For the convenience of the experiments, one slot duration is assumed to be equal to the transmission time of one packet at $r_{1}$ under stable environment. In each experiment, we assume that each sender of the randomly selected links has 200 packets to transmit. We record the completion time and calculate the corresponding 


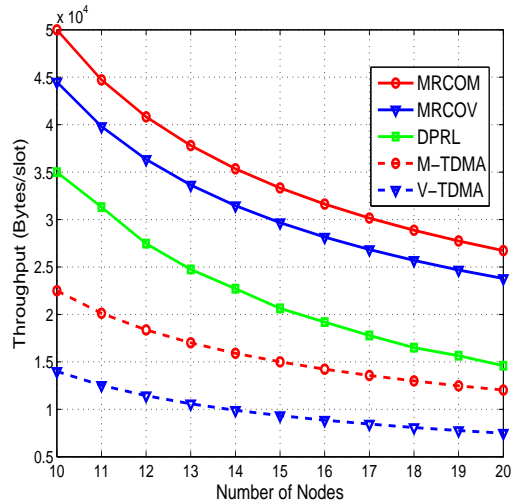

Fig. 3: The throughput comparison of MTDMA, V-TDMA, MRCOM, MRCOV and DPRL for different node densities.

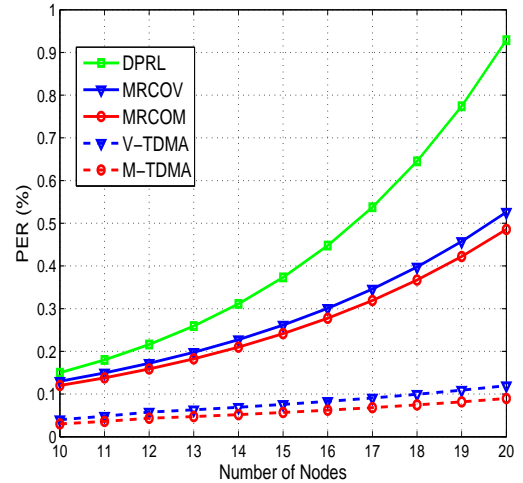

Fig. 4: PER comparison of M-TDMA, VTDMA, MRCOM, MRCOV and DPRL for different node densities.

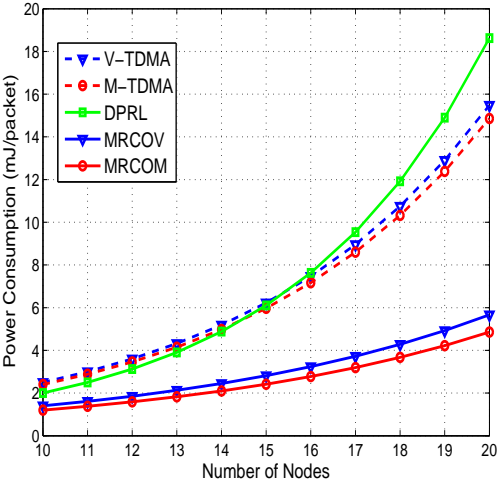

Fig. 5: Power consumption comparison of M-TDMA, V-TDMA, MRCOM, MRCOV and DPRL for different node densities.

throughput, PER, and power consumption for transmitting all packets. Each data point is averaged over 300 simulation runs.

Fig. 3 shows the per link throughput attained under MRCOM, MRCOV, standard M-TDMA, V-TDMA and DPRL. The figure shows that, the throughput under MRCOM, MRCOV and DPRL are much higher than those attained by M-TDMA and V-TDMA since multiple simultaneous transmissions are possible in all scenarios. At the same time, MRCOM and MRCOV both outperform DPRL following our mobility pattern based link selection, data rate and power level assignment mechanism. Further, the throughput of all algorithms almost monotonically decreases as the number of nodes increases. The main reason is that the possibility of links move through each other' effective interference range increases as the increasing of the node density. Due to the dogmatic mechanism used by DPRL, its throughput decreases much sharper than MRCOM and MRCOV. It must be noted that the finish time of data packets is an important performance measurement for delay-sensitive applications. To that end, the per link throughput reflects the delay performance of all algorithms under our simulation settings. In other words, the reciprocal of per link throughput represents the delay performance.

Fig. 4 shows the average PER corresponding to Fig. 3. As depicted, the general trend of all the algorithm's PER increase with the increasing of the node density. The main reason is that the increasing intensive interactive movements increase the collisions under all the simultaneous transmission scenarios. The other reason is that higher density boosts the average number of links in one successful simultaneous transmission scenario. Therefore, the mutual interference in a successful simultaneous transmission scenario is increased. Further and due to interference caused by simultaneous transmissions, the PER of MRCOM and MRCOV are both higher than their associated original protocols. Due to the static working mode, the performance of DPRL deteriorates significantly as the increasing of the node density. Since the optimal link selection mechanism and the dynamic mode changing minimizes the mutual interference of the simultaneous transmissions, the PER of MRCOM and MRCOV are both much lower than that of DPRL.

Fig. 5 depicts the average power consumption of transmitting one packet under different algorithms. Although, MRCOM and MRCOV are both simultaneous transmission environments, the power consumption of MRCOM and MRCOV remain much lower than that of M-TDMA and V-TDMA in all scenarios. That is because our dynamic mode changing mechanism not only minimizes interference between simultaneous transmissions, but also improves the throughput significantly in all scenarios. As depicted, the power consumption of DPRL is much higher than that of MRCOM and MRCOV. This is corresponding to the fact that high mutual interference in DPRL increases transmission collisions significantly and the retransmissions waste a lot of energy. 


\section{Conclusions}

In this paper, we proposed a mobility pattern adaptive resource combinatorial optimization algorithm as a generic STDMA strategy to dynamically generate optimal simultaneous transmission schedules. Our low complexity MPARCO algorithm can minimize the energy consumption under weak mobility scenarios and maximize the throughput capacity under strong mobility scenarios. We compared the performance of our proposed algorithm to that of original M-TDMA, V-TDMA and DPRL algorithms and showed that our algorithm can significantly improve the throughput and energy consumption performance of existing mobility-aware STDMA MAC protocols. Currently, we are in process of extending our work to cover propagation models accounting for time-varying fading channels and offering priority mechanisms of accommodating transmitting nodes.

\section{References}

[1]A. Jhumka and S. Kulkarni, "On the design of mobility-tolerant tdma based media access control (mac) protocol for mobile sensor networks," in Proc. 4th international conference on Distributed computing and internet technology, Vol. 4882, pp. 42-53, 2007.

[2] Rashid A. Saeed and Mahamat A. Abakar, "Design and evaluation of lightweight IEEE80211p-based TDMA MAC method for road side-to-vehicle communications," in Proc. International Conferenceon Computer and Communication Engineering, pp. 1-5, 2010

[3] C. Schindelhauer, "Mobility in wireless networks," in SOFSEM, pp. 100-116, 2006.

[4] A. Raja and X. Su, "Mobility handling in mac for wireless ad hoc networks," Wireless Communications and Mobile Computing, Vol. 9, pp. 303C311, March 2009.

[5] T. Elbatt and A. Ephremides, "Joint Scheduling and Power Control for Wireless Ad Hoc Networks," IEEE Trans. Wireless Commun., Vol. 3, no. 1, pp. 74-85, Jan. 2004.

[6] M. Zafer, E. Modiano, "Optimal adaptive data transmission over a fading channel with deadline and power constraints," in Proc. Annu. Conf. Inform. Sci. Syst., pp. 931-937, 2006.

[7] J. Kivinen, X. Zhao, and P. Vainikainen, "Empirical characterization of wideband indoor radio channel at 5.3 Ghz," IEEE Trans. Antennas Propag., Vol. 49, no. 8, pp. 1192-1203, Aug. 2001.

[8] H. Yousefi'zadeh, H. Jafarkhani, M. Moshfeghi, "Power Optimization of Wireless Media Systems with Space-Time Block Codes," IEEE Trans. on Image Processing, July 2004.

[9] H. Yousefi'zadeh, H. Jafarkhani, "An Optimal Power-Throughput Tradeoff Study for MIMO Fading Ad-Hoc Networks," KICS/IEEE J. of Communications and Networks (JCN), Invited Paper, Aug. 2010. [10] F. Bai, N. Sadagopan, A. Helmy, "A Framework to Systematically Analyze the Impact of Mobility on Performance of Routing Protocols for Adhoc NeTworks," in Proc. IEEE INFOCOM, 2003. 